Leonardo FAVILLI*

\title{
Osservazioni sulla fauna a Lepidotteri Ropaloceri delle Isole Eolie (Sicilia) (Lepidoptera: Papilionoidea)
}

\begin{abstract}
Riassunto: Nel 2014 sono stati raccolti dati su alcune specie di Ropaloceri delle Isole Eolie (Sicilia, Italia). Questi dati precisano meglio la distribuzione di otto specie e nel caso di Cacyreus marshalli Butler, [1898] e di Danaus chrysippus (Linné, 1758) costituiscono la prima segnalazione per queste isole.
\end{abstract}

\begin{abstract}
Observations on the butterfly fauna (Rhopalocera) of the Aeolian Islands (Sicily) (Lepidoptera: Papilionoidea). In year 2014 we collected many records of Rhopalocera of Aeolian Islands (Sicily, Italy). These records contribute to the distribution of eight species and in case of Cacyreus marshalli Butler, [1898] and Danaus chrysippus (Linné, 1758) are the first reports from the Archipelago.
\end{abstract}

Key words: Butterflies, Aeolian Islands (Sicily, Italy), new records, checklist.

\section{INTRODUZIONE}

Nella presente nota vengono riportati i dati relativi a otto specie di Ropaloceri nuove per l'arcipelago delle Eolie o per almeno una delle sue isole, rilevate durante una serie di visite effettuate tra il $6 \mathrm{e}$ il 12 settembre 2014 con l'intento di aggiornare il quadro delle conoscenze sulla fauna a Lepidotteri di questa parte della Sicilia insulare.

\section{MATERIALI E METODI}

Per ogni specie viene fornita una scheda sintetica con elencati: i materiali esaminati, indicando i toponimi delle località (tratti dalla Carta Topografica d'Italia dell'IGMI scala 1:25.000 Serie M 891), l'altitudine, il comune e la provincia, il rilevatore, la data e il numero di esemplari osservati (tra parentesi); una serie di osservazioni relative al motivo di interesse della segnalazione e alla presenza della specie in Sicilia.

Per la tassonomia e la nomenclatura si è fatto riferimento a Balletto et al. (2014).

Il riconoscimento specifico è stato effettuato direttamente a vista o dopo la cattura eseguita ricorrendo a retini entomologici. Tutti gli esemplari catturati, una volta determinati, sono stati liberati.

\section{ELENCO DELLE SPECIE}

Pieris edusa (Fabricius, 1777) (Pieridae)

Materiali esaminati. Salina, Malfa, $50 \mathrm{~m}$ (Malfa,
Messina), L. Favilli leg. 11.IX.2014 (3 esemplari); Salina, Pollara, $60 \mathrm{~m}$ (Malfa, Messina), L. Favilli leg. 11.IX.2014 (2 esemplari).

OSSERVAZIONI. P. edusa è nuova per Salina; è stata rilevata lungo la strada che da Malfa conduce a Punta di Scario e in una zona a macchia mediterranea poco a monte della spiaggia di Pollara. Questa specie era già nota per le Isole Eolie, essendo stata segnalata per Lipari, Stromboli e Vulcano (Tab. 1). In Sicilia è ampiamente diffusa ed è conosciuta anche per Lampedusa, Pantelleria e Levanzo (Balletto et al., 2007).

Pieris rapae (Linné, 1758) (Pieridae)

Materiali esaminati. Salina, Santa Marina Salina, 25 m (Santa Marina Salina, Messina), L. Favilli leg. 11.IX.2014 (5 esemplari).

OSSERVAZIONI. P. rapae è nuova per Salina; è stata rilevata nel tratto della S.P. 182 sovrastante il porto, in un'area caratterizzata da insediamenti abitativi, con giardini e appezzamenti incolti. Questa specie era già nota per le Isole Eolie, essendo stata segnalata per Lipari, Stromboli e Vulcano (Tab. 1). In Sicilia è molto diffusa ed è conosciuta anche per Lampedusa, Pantelleria, Favignana e Levanzo (Balletto et al., 2007).

Leptotes pirithous (Linné, 1767) (Lycaenidae) MATERiali eSAminati. Vulcano, Vulcanello, $125 \mathrm{~m}$ (Lipari, Messina), L. Favilli leg. 10.IX.2014 (2 esem-

*Leonardo Favilli, Dipartimento di Scienze Fisiche della Terra e dell'Ambiente, Via Mattioli 4, 53100 Siena (SI), Italia. E-mail: leonardo.favilli@unisi.it 
plari); pendici del Gran Cratere, 140 m (Lipari, Messina), L. Favilli leg. 10.IX.2014 (3 esemplari).

OSSERVAZIONI. L. pirithous è nuovo per Vulcano; è stato rilevato in un'area a macchia bassa e ga- riga sulle pendici di Vulcanello e in una gariga lungo il sentiero che porta al Gran Cratere. Questa specie era già nota per le Isole Eolie, essendo stata segnalata per Lipari e Stromboli (Tab. 1). In Sicilia è abbastanza dif-

Tab. 1. Checklist dei Ropaloceri delle Isole Eolie. • dati ripresi da Kudrna \& Leigheb (1988), Biermann (2005) e Balletto et al. (2007); $\boldsymbol{\Delta}$ specie segnalata per la prima volta in questa nota.

\begin{tabular}{lcccc}
\hline Specie & Isola & & \\
& Lipari & Salina Vulcano Stromboli Filicudi Alicudi Panarea
\end{tabular}

\section{Famiglia Hesperiidae}

1) Gegenes nostrodamus (Fabricius, 1793)

2) Gegenes pumilio (Hoffmannsegg, 1804)

$\begin{array}{lllllll}- & - & \bullet & - & - & - & - \\ - & - & - & - & - & - & -\end{array}$

\section{Famiglia Papilionidae}

3) Papilio machaon Linné, 1758

\section{Famiglia Pieridae}

4) Pieris brassicae (Linné, 1758)

5) Pieris edusa (Fabricius, 1777)

6) Pieris mannii (Mayer, 1851)

7) Pieris napi (Linné, 1758)

8) Pieris rapae (Linné, 1758)

9) Colias crocea (Geoffroy, 1785)

\section{Famiglia Lycaenidae}

10) Lycaena phlaeas (Linné, 1761)

11) Leptotes pirithous (Linné, 1767)

12) Cacyreus marshalli Butler, [1898]

13) Lampides boeticus (Linné, 1767)

14) Celastrina argiolus (Linné, 1758)

15) Pseudophilotes baton (Bergsträsser, 1779)

16) Aricia agestis ([Denis \& Schiffermüller], 1775)

17) Polyommatus icarus (Rottemburg, 1775)

\section{Famiglia Nymphalidae}

18) Vanessa atalanta (Linné, 1758)

19) Vanessa cardui (Linné, 1758)

20) Polygonia egea (Cramer, [1775])

21) Charaxes jasius (Linné, 1767)

22) Hipparchia leighebi Kudrna, 1976

23) Maniola jurtina (Linné, 1758)

24) Pyronia cecilia (Vallantin, 1894)

25) Pararge aegeria (Linné, 1758)

26) Lasiommata megera (Linné, 1758)

$\begin{array}{llllll}\bullet & \bullet & \bullet & \bullet & - & - \\ \bullet & \Delta & \bullet & \bullet & - & - \\ \bullet & \bullet & \bullet & \bullet & - & - \\ \bullet & \bullet & \bullet & \bullet & - & - \\ \bullet & \Delta & \bullet & \bullet & - & - \\ \bullet & \bullet & \bullet & \bullet & - & -\end{array}$

\section{Famiglia Danaidae}

7) Danaus chrysippus (Linné, 1758)

$\mathrm{N}^{\circ}$ totale di specie

24

19

23

13

$\mathbf{0}$

0

1 
fusa ed è conosciuta anche per Pantelleria e Marettimo (Balletto et al., 2007).

Cacyreus marshalli Butler, [1898] (Lycaenidae) MATERIALi esAminati. Lipari, Lipari, 20 m (Lipari, Messina), L. Favilli leg. 06.IX.2014 (8 esemplari); Lipari, Canneto, $3 \mathrm{~m}$ (Lipari, Messina), L. Favilli leg. 06.IX.2014 (5 esemplari); Salina, Santa Marina Salina, 20 m (Santa Marina Salina, Messina), L. Favilli leg. 11.IX.2014 (1 esemplare).

Osservazioni. C. marshalli è nuovo per le Isole Eolie; è stato rilevato in volo e posato su fioriere di pelargoni nei centri abitati di Lipari, di Canneto e di Santa Marina Salina. Questa specie è nota per varie località della Sicilia ma fino ad oggi non era mai stata segnalata in alcuna delle sue isole (Balletto et al., 2007). ऐ̀ possibile che sia comparso a Lipari introdotto passivamente con pelargoni infestati provenienti dalla $\mathrm{Si}$ cilia o da altre località italiane. Trattandosi di un'entità in rapida espansione in Italia, è molto probabile che sia in grado di acclimatarsi a Lipari e anche a Salina e che possa colonizzare anche qualcuna delle altre isole dell'arcipelago.

Lampides boeticus (Linné, 1767) (Lycaenidae) Materiali esaminATI. Lipari, Bagni Termali S. Calogero, $140 \mathrm{~m}$ (Lipari, Messina), L. Favilli leg. 08.IX.2014 (4 esemplari).

OSSERVAZIONI. L. boeticus è specie nuova per Lipari; è stato rilevato tra la vegetazione ruderale che cresce attorno all'antica vasca termale. Questa specie era già nota per l'arcipelago, essendo stata segnalata per Salina e Vulcano (Tab. 1). In Sicilia è abbastanza diffusa, ma a parte le Eolie non è conosciuta nelle altre isole circumsiciliane (Balletto et al., 2007).

\section{Vanessa cardui (Linné, 1758) (Nymphalidae)}

Materiali esaminati. Lipari, Campo Bianco, $60 \mathrm{~m}$ (Lipari, Messina), L. Favilli leg. 08.IX.2014 (3 esemplari); Vulcano, Sorgenti Termali, 2 m (Lipari, Messina), L. Favilli leg.10.IX.2014 (1 esemplare).

OsSERVAZIONI. V. cardui è nuova per Lipari e per Vulcano; nella prima è stata rilevata tra gli edifici della ex zona industriale delle cave di pomice di Campo Bianco, lungo la strada che da Canneto conduce a Porticcioli mentre a Vulcano è stata osservata tra le rocce del sito termale. Questa specie era già nota per le Isole Eolie, essendo stata segnalata per Salina e Stromboli (Tab. 1). In Sicilia è ampiamente diffusa ed è cono- sciuta anche per Lampedusa, Linosa, Pantelleria, Marettimo e Levanzo (Balletto et al., 2007). Trattandosi di una specie euricora, molto vagile e migratrice (Balletto \& Kudrna, 1985), era logico attendersi che fosse presente anche a Lipari e a Vulcano.

\section{Charaxes jasius (Linné, 1767) (Nymphalidae)}

Materiali esaminati. Lipari, Forgia Vecchia, 285 m (Lipari, Messina), L. Favilli leg. 12.IX.2014 (2 esemplari).

OSSERVAZIONI. C. jasius è specie nuova per Lipari; è stato rilevato in volo in un'area a macchia a mediterranea con presenza di corbezzolo (Arbutus unedo), pianta ospite delle larve. Questa specie era già nota per le Isole Eolie, essendo stata segnalata per Salina (Tab. 1). In Sicilia è discretamente diffusa, ma a parte le Eolie non è conosciuta nelle altre isole circumsiciliane (Balletto et al., 2007). Trattandosi di una specie molto vagile (Balletto \& Kudrna, 1985) era plausibile fosse presente anche a Lipari dove il corbezzolo è ben diffuso.

Danaus chrysippus (Linné, 1758) (Danaidae) MATERIALI ESAMINATI. Vulcano, Gran Cratere, presso la vetta, $300 \mathrm{~m}$ (Lipari, Messina), L. Favilli leg. 10.IX.2014 (1 esemplare).

OsSERVAZIONI. D. chrysippus è nuovo per le Isole Eolie; è stato rilevato posato sulle rocce nude che si trovano presso la vetta del Gran Cratere. Si tratta di una specie a corotipo afrotropicale-indiano-mediterraneo in espansione in Europa meridionale in seguito al riscaldamento climatico che ha interessato negli ultimi anni il bacino occidentale del Mediterraneo (Balletto et al., 2009). A causa di ciò è stata segnalata in un buon numero di regioni italiane, in particolare in quelle meridionali, incluse varie località della Sicilia comprese Linosa e Lampedusa (Balletto et al., 2007; Pisciotta et al., 2008; Viviano \& Viviano, 2016). Nei prossimi anni sarà interessante monitorare la presenza di questa specie nelle Isole Eolie per verificare se si è trattato di un fatto occasionale dovuto a un esemplare in migrazione o se esiste la possibilità che si instaurino colonie stabili a Vulcano o in qualche altra isola dell'arcipelago.

\section{CONCLUSIONI}

Con i dati presentati in questa nota vengono aggiunte quattro specie a quelle note per Lipari e tre a quelle conosciute per Salina e per Vulcano e viene portato a 27 il numero di Ropaloceri segnalati fino a oggi nell'arcipelago (Tab. 1). 
Alla lista potrebbe essere aggiunta Libythea celtis (Laicharting, [1782]), segnalata da Tolman \& Lewington (1997) per Lipari, ma la sua presenza nell'isola non è stata riportata dagli autori successivi (Parenzan \& Porcelli, 2006; Balletto et al., 2007) ed è riportata con dubbio da Biermann (2005). Questa specie, tuttavia, mostra tendenze dispersive ed è possibile che durante i suoi spostamenti possa comparire in qualcuna delle Isole Eolie.

Con esclusione di C. marshalli, come già ricordato con ogni probabilità introdotto passivamente dall'uomo, tutti i Ropaloceri campionati sono molto vagili (Balletto \& Kudrna, 1985), caratteristica che ha favorito la loro dispersione da un'isola all'altra del- l'arcipelago o dalle prospicienti coste siciliane. Si ritiene che nuove ricerche possano incrementare il catalogo faunistico; in particolare sarà senz'altro possibile aumentare il numero di specie conosciute per Panarea (ad oggi una sola) e trovarne qualcuna ad Alicudi e Filicudi (nessuna ad oggi nota). La pressoché totale assenza di Ropaloceri in queste isole, infatti, più che da effettiva povertà faunistica, dipende con molte probabilità dal fatto che ad esse è stata dedicata minore attenzione da parte degli entomologi di quanto è stato fatto per le altre isole dell'arcipelago a causa della difficile accessibilità dovuta alla scarsa presenza di collegamenti marittimi, all'aspra morfologia e alla limitata viabilità interna.

\section{BIBLIOGRAFIA}

Balletto E., Barbero F., Casacci L.P., Cerrato C., Patricelli D., Bonelli S., 2009 - L'impatto dei cambiamenti climatici sulle farfalle italiane. Studi Trentini di Scienze Naturali, 86: 111-114.

Balletto E., Bonelli S., Cassulo L., 2007 - Insecta Lepidoptera Papilionoidea (Rhopalocera). In: Ruffo S., Stoch F. (eds.), Checklist and distribution of the Italian fauna. Memorie del Museo civico di Storia naturale di Verona, 2. serie, Sezione Scienze della vita, 17 (2006): 257-261, with data on CD-ROM.

Balletto E., Cassulo L., Bonelli S., 2014 - Annotated checklist of the italian butterflies and skippers (Papilionoidea, Hesperiioidea). Zootaxa, 3853: 1-114.

Balletto E., KudRna O., 1985 - Some aspects of the conservation of butterflies in Italy, with recommendations for a future strategy (Lepidoptera Hesperiidae \& Papilionoidea). Bollettino della Società Entomologica Italiana, 117: 39-59.

BIERMANN H., 2005 - Schmetterlinge der Liparischen Inseln (Lepidoptera, Rhopalocera). Atalanta, 36 (1/2): 113-118.

KudRna O., Leigheb G., 1988 - On the butterflies (Lepidoptera: Rhopalocera) of some Tyrrhenian islands (southern Italy). British Journal of Entomology and Natural History, 1: 133-137.

PAREnZAN P., Porcelli F., 2006 - I macrolepidotteri italiani. Fauna Lepidopterum Italiae (Macrolepidoptera). Phytophaga, 15: $1-1051$.

Pisciotta S., Zito P., SaJeva M., 2008 - Danaus chrysippus (Linnaeus, 1758) (Lepidoptera, Nymphalidae) larvae feeding on Caralluma europaea (Guss.) N.E.BR. (Asclepiadacee) in Lampedusa Island. Il Naturalista Siciliano Serie IV, 1/2: 241251

Tolman T., Lewington R., 1997 - Collins field guide Butterflies of Britain \& Europe. Harper Collins, 350 pp.

Viviano R., Viviano A., 2016 - New data on Coleoptera species in Natura Reserve "Torre Salsa" (Sicily, Italy). Biodiversity Journal, 7 (4): 945-950. 